\title{
M3FT-15OR0202237: SUBMIT REPORT ON RESULTS FROM INITIAL COATING LAYER DEVELOPMENT FOR UN TRISO PARTICLES
}

\author{
Brian Jolly ${ }^{1}$, Terrance Lindemer ${ }^{2}$, Kurt Terrani ${ }^{1}$ \\ ${ }^{1}$ Oak Ridge National Laboratory, Oak Ridge, TN, 37831-6063 \\ ${ }^{2}$ Harbach Engineering and Solutions, Dayton, Ohio 45458 \\ jollybc@ornl.gov
}

\section{INTRODUCTION}

In support of fully ceramic matrix (FCM) fuel development [1-2], coating development work has begun at the Oak Ridge National Laboratory (ORNL) to produce tri-isotropic (TRISO) coated fuel particles with UN kernels. The nitride kernels are used to increase heavy metal density in these SiC-matrix fuel pellets with details described elsewhere [3]. The advanced gas reactor (AGR) program at ORNL used fluidized bed chemical vapor deposition (FBCVD) techniques for TRISO coating of UCO (two phase mixture of $\mathrm{UO}_{2}$ and $U C_{x}$ ) kernels [4]. Similar techniques were employed for coating of the UN kernels, however significant changes in processing conditions were required to maintain acceptable coating properties due to physical property and dimensional differences between the UCO and UN kernels (Table 1).

\section{Table 1: Comparison of UCO and UN kernel properties}

\begin{tabular}{|l|l|l|}
\hline & AGR-1 (UCO) & UN kernels \\
\hline Nominal diameter $(\mu \mathrm{m})$ & 350 & 800 \\
\hline Density $\left(\mathrm{g} / \mathrm{cm}^{3}\right)$ & 10.92 & 14 \\
\hline Specific heat $[\mathrm{J} / \mathrm{g}-\mathrm{K}]$ & $>0.3$ & 0.25 \\
\hline Thermal conductivity $(\mathrm{W} / \mathrm{mK})$ & $<5$ & $15.5^{*}$ \\
\hline
\end{tabular}

* Literature values at $1000{ }^{\circ} \mathrm{C}$. Actual values have not been measured.

\section{Experimental Procedures}

The FBVCD coating furnace has been refurbished and critical temperature and gas delivery systems have been calibrated. An initial "shake down" coating experiment has been completed with equipment performing well. Early indications show significant increases $(\sim 50 \%)$ in gas flows are required to fluidize the UN kernels compared to the UCO kernels previously used. Additional information on UN kernel coating behavior will be available in the coming months as coating development progresses.

\section{Equipment}

The coating furnace (Figure 1) consists of a conical graphite coating chamber surrounded by a resistively heated graphite element which are both housed within a 
water cooled shell. Process gases are delivered through a water cooled injector which supports the coating chamber. Kernels are loaded through the top of the furnace at room temperature with argon gas flowing through the injector. The furnace is brought to coating temperature and the individual layers are deposited by switching between process gasses. Once the coating experiment is complete, the furnace is brought back to room temperature and gas flow is shut off allowing the kernels to drain through the injector and into the catch cup fixed to the bottom of the injector.

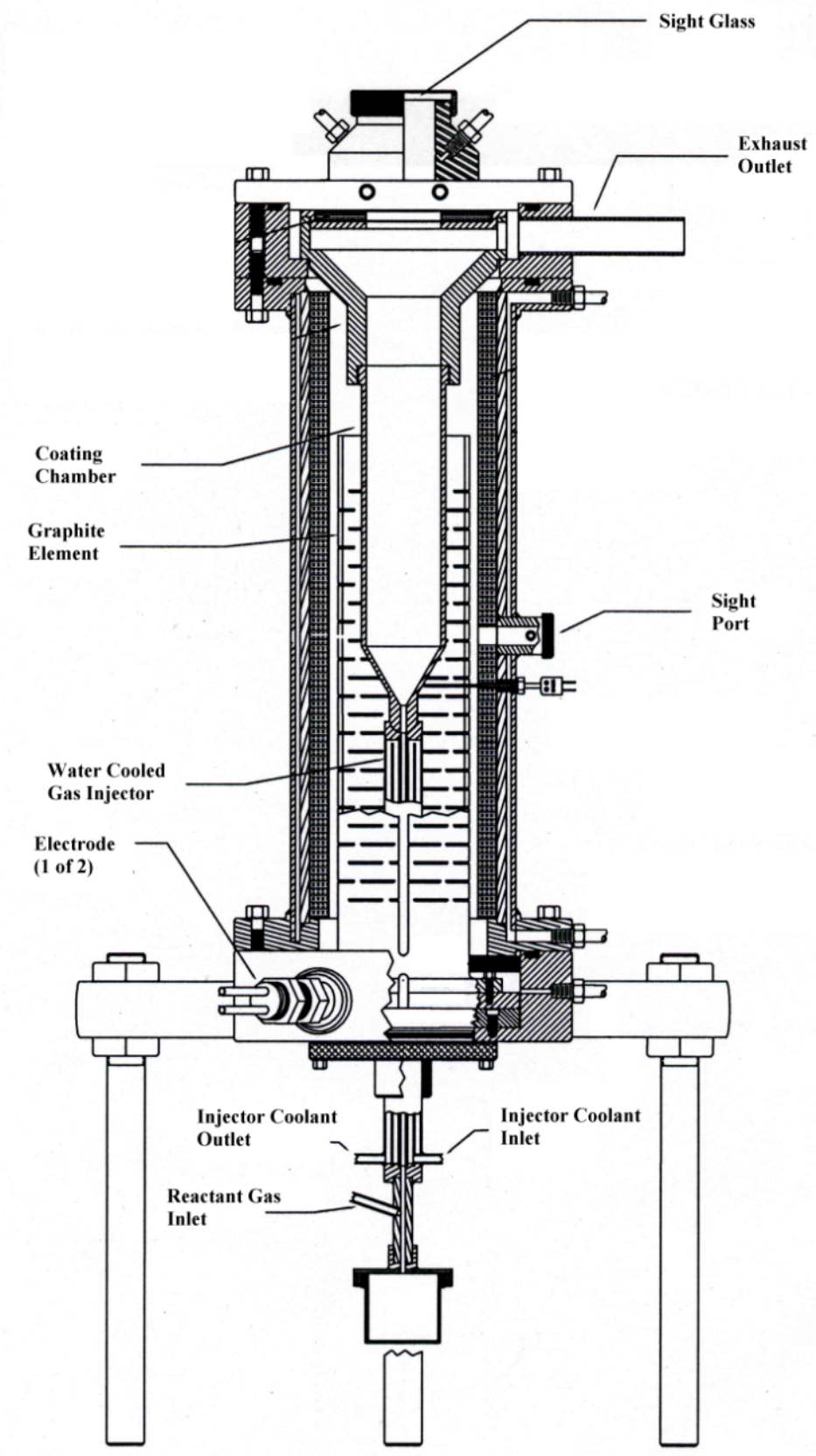

Figure 1: Schematic of FBCVD coating furnace used for TRISO coating of UN kernels 
Temperature of the fluidized bed is measured directly with a hand held optical pyrometer which looks though the top of the furnace via a sight glass and prism. Signal attenuation from the sight glass/prism set is measured and the appropriate correction factor applied. Gas delivery is controlled using electronic mass flow controllers (MFC). The MFCs are periodically calibrated using a wet test meter.

\section{RESULTS AND DISCUSSION}

\section{Initial processing conditions and batch size}

Coating temperature and concentration of reactants play dominant rolls in controlling the properties of deposited layers. Initially reactant concentration to the surface (flux) will be held constant and temperature will be varied to attain the desired coating properties.

For this work, batch size is determined by the surface area of the kernels, rather than weight, as surface area governs flux for a given coating gas fraction (CGF). CGF is defined as precursor gas flow divided by total gas flow. During the AGR program, batch surface area was $\sim 1000 \mathrm{~cm}^{2}$. For this work, batch size was selected to be $\sim 500 \mathrm{~cm}^{2}$ in order to maintain a reasonable batch volume given the large increase in kernel diameter. This means that the CGF will be half that used during the AGR program for UCO kernels, implying flux of reactants to the surface will be similar.

Properties for each layer of the TRISO system are dependent upon the properties of the preceding layer. So, coating development will proceed in a stepwise fashion with processing conditions for the buffer layer being optimized before proceeding to the inner pyrocarbon layer (IPyC), and processing conditions for the IPyC layer being optimized before beginning $\mathrm{SiC}$ depositions etc. This progressive strategy will be maintained throughout the UN TRISO development process. However, for the first coating experiment all four layers were deposited. This was done primary to ensure for full functionality of the coating equipment, but it also helped to provide a quick assessment to check for unanticipated problems.

\section{Initial results}

On average, the first coating experiment went well. The temperature control and gas delivery systems performed as expected and all four coating layers were successfully deposited. Figure 2 shows optical micrographs of seven coated particles randomly selected from the large batch of coated particles. These particles were cross sectioned and polished to mid-plane for thickness and microstructural analysis. A higher magnification image of particles 3 and 7 (Figure 3) shows the deposited layers in greater detail. While the properties of the buffer, IPyC, and OPyC (outer pyrocarbon) layers will almost certainly have to be adjusted to meet specifications during the coating development process, for a first approximation the microstructural appearance of each layer is consistent with coatings deposited during the AGR program. 
The area that needs the most significant improvement after this first coating experiment was the microstructure of the $\mathrm{SiC}$ layer. Looking at Figure 3, numerous soot inclusions can be seen within the SiC layer and the outer surface appears nodular. These features are most likely due to the kernels being under-fluidized during the $\mathrm{SiC}$ deposition stage. Under-fluidized kernels can become stagnant and dwell too long on the coating chamber walls causing carbon soot from the previous coating steps to become trapped within the $\mathrm{SiC}$ layer. Also, it was discovered at the end of the coating experiment that numerous kernels had become stuck to the coating chamber walls. These kernels had only $\mathrm{SiC}$ deposited on them, with no OPyC present, indicating that they had become stuck during the $\mathrm{SiC}$ deposition process; this is another known symptom of underfluidization. In fact, from visual inspection of the fluidized particle bed during $\mathrm{SiC}$ coating, it was noted that increased gas flow rates to improve fluidization was needed. However, the flow meter was already operating at its maximum capacity. A higher capacity flow meter will be installed prior to the next coating experiment so that proper fluidization can be attained.

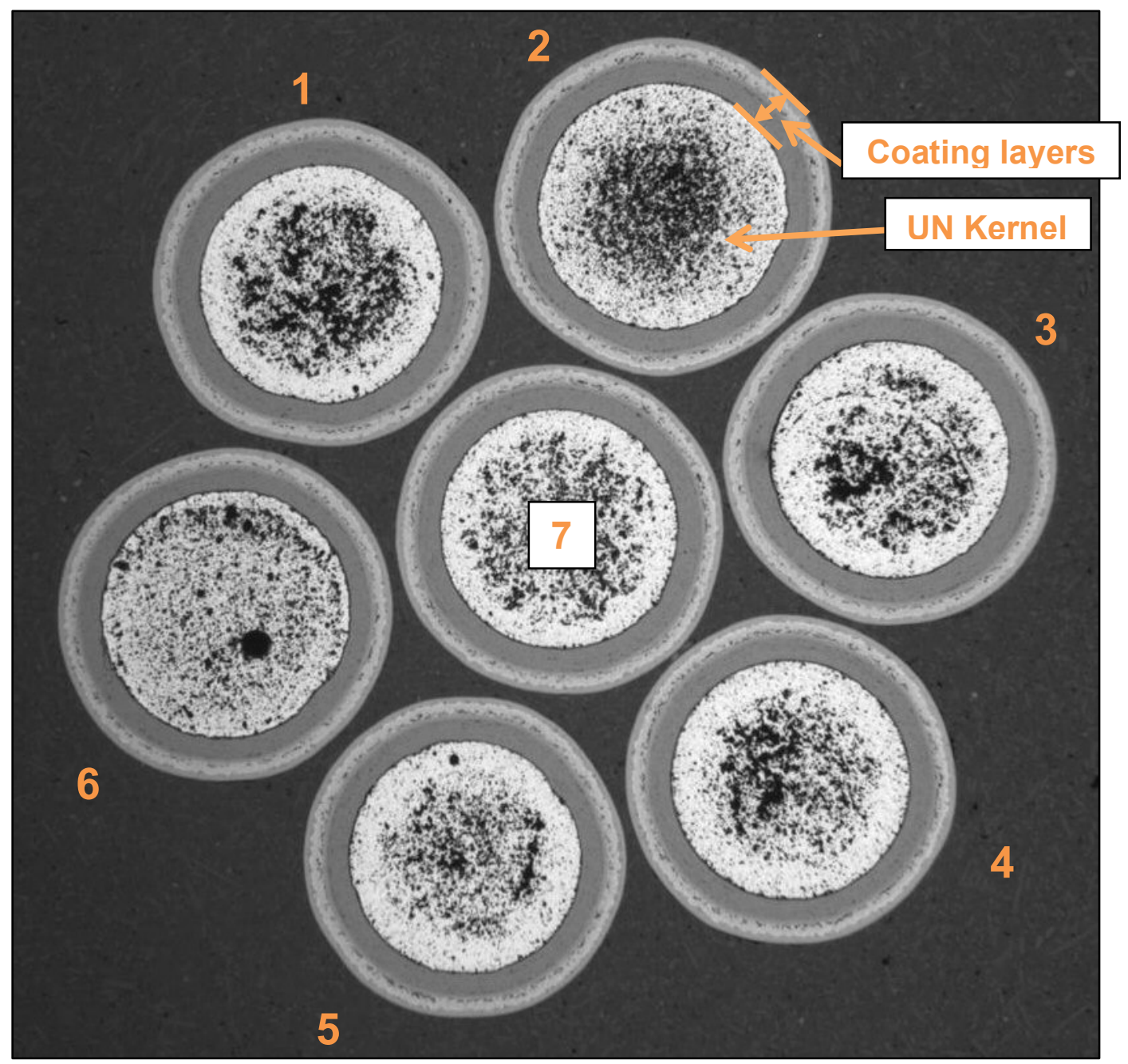

Figure 2: 7 coated UN kernels mounted and polished to mid-plane for microstructural examination and coating thickness measurement. The particles were arbitrarily numbered for reference. 


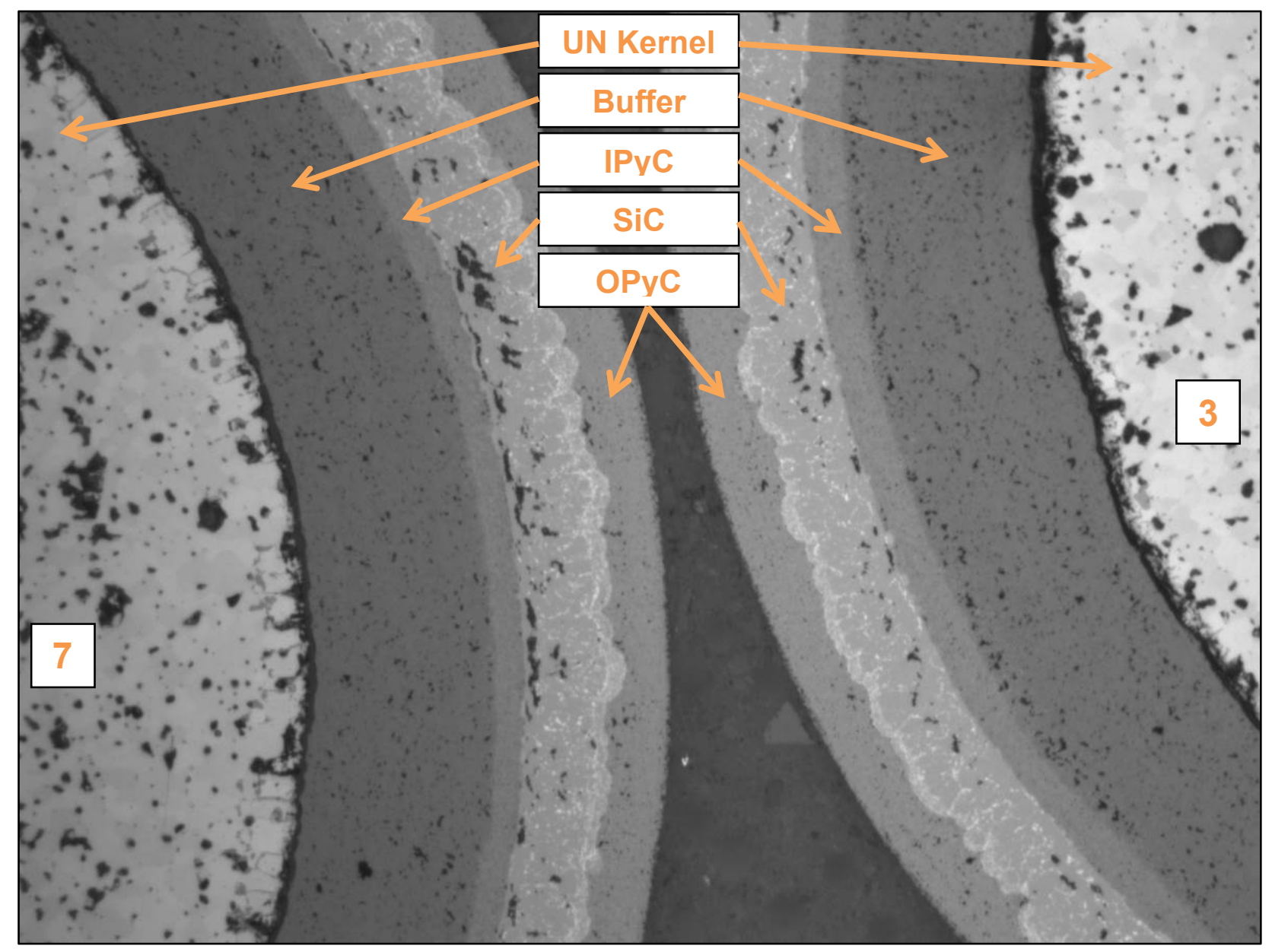

Figure 3: Higher magnification image of coated UN kernels number 3 and 7.

Thickness measurements for each coating layer were made on particles 1,4 , and 7 (Table 2). These measurements provide a quick estimate of the coating rate for each layer and will be used as guidance to adjust thickness for future coating experiments. Controlling coating thickness is fairly straight forward as it is primarily a function of coating duration. The thickness of the various layers achieved after this first coating trial is consistent with the desired target except for the IPyC layer.

Table 2: Coating thickness results from initial coating experiment.

\begin{tabular}{|c|c|c|c|c|c|}
\hline & Target & Particle 1 & Particle 4 & Particle 7 & $\begin{array}{c}\text { Average of 3 } \\
\text { particles }\end{array}$ \\
\hline $\begin{array}{c}\text { Kernel (radius) } \\
(\mu \mathrm{m})\end{array}$ & & 432 & 429 & 428 & 430 \\
\hline Buffer $(\mu \mathrm{m})$ & 75 & 84 & 72 & 78 & 78 \\
\hline $\mathrm{IPyC}(\mu \mathrm{m})$ & 30 & 16 & 13 & 12 & 14 \\
\hline $\mathrm{SiC}(\mu \mathrm{m})$ & 35 & 39 & 40 & 46 & 42 \\
\hline OPyC $(\mu \mathrm{m})$ & 30 & 28 & 27 & 27 & 27 \\
\hline
\end{tabular}




\section{SUMMARY}

A coordinated effort is underway to produce TRISO coated fuel particles from recently processed uranium mononitride kernels at ORNL. These particles will be used in subsequent irradiation tests of the FCM fuel concept under prototypic LWR conditions.

Early progress toward coating development for these kernels has proved to be successful. The FBCVD coating furnace has been successfully refurbished and an initial coating experiment, where all four layers were deposited, has been performed, confirming the functionality of the furnace and associated equipment. Now that the ground work has been laid, future coating development will focus on optimizing the properties of each coating layer to meet fuel specification.

\section{ACKNOWLEDGMENTS}

Chinthaka Silva and John Henry Jr. at ORNL contributed to the effort. The work presented in this transaction was supported by the Advanced Fuels Campaign of the Fuel Cycle R\&D program in the Office of Nuclear Energy, US Department of Energy.

\section{REFERENCES}

[1] L. L. Snead; K. A. Terrani; F. Venneri; Y. Kim; J. E. Tulenko; C. W. Forsberg, et al., Transactions of the American Nuclear Society 2011, 104.

[2] K. A. Terrani; L. L. Snead; J. C. Gehin, Journal of Nuclear Materials 2012, 427 (1-3), 209-224.

[3] N. M. George; I. Maldonado; K. A. Terrani; A. Godfrey; J. Gehin; J. Powers, Nuclear Technology 2014, 188 (3).

[4] J. D. Hunn; R. A. Lowden Data compilation for AGR-1 baseline coated particle composite LEU01-46T, ORNL/TM-2006/019 Oak Ridge National Laboratory: 2006.

Notice: This manuscript has been authored by UT-Battelle, LLC, under Contract No. DE-AC05-00OR22725 with the U.S. Department of Energy. The United States Government retains and the publisher, by accepting the article for publication, acknowledges that the United States Government retains a non-exclusive, paid-up, irrevocable, world-wide license to publish or reproduce the published form of this manuscript, or allow others to do so, for United States Government purposes. 